\title{
Diacronie
}

Studi di Storia Contemporanea

$\mathrm{N}^{\circ} 11,3$ | 2012

La satira fa storia. Eventi, pratiche, linguaggi

\section{Elena Aga Rossi, Maria Teresa Giusti, Una guerra a parte. I militari italiani nei Balcani 1940-1945}

\section{Mario De Prospo}

\section{(e) OpenEdition}

Edizione digitale

URL: http://journals.openedition.org/diacronie/2732

DOI: 10.4000/diacronie.2732

ISSN: 2038-0925

Editore

Association culturelle Diacronie

Notizia bibliografica digitale

Mario De Prospo, «Elena Aga Rossi, Maria Teresa Giusti, Una guerra a parte. I militari italiani nei Balcani 1940-1945 », Diacronie [Online], № 11, 3 | 2012, documento 14, Messo online il 29 octobre 2012,

consultato il 23 septembre 2020. URL : http://journals.openedition.org/diacronie/2732 ; DOI : https:// doi.org/10.4000/diacronie.2732 


\section{Diacronie}

N. 11 | 3|2012 La satira fa storia. Eventi, pratiche, linguaggi

14/

\section{RECENSIONE:}

\section{Elena AGA ROSSI, Maria Teresa GIUSTI, Una guerra a parte. I militari italiani nei Balcani 1940-1945, Bologna, Il Mulino, 2011, 660 pp.}

a cura di Mario DE PROSPO *

Elena Aga Rossi e Maria Teresa Giusti sono due studiose che sulle vicende dei militari italiani nel corso dell'ultimo conflitto mondiale hanno già avuto modo di fornire considerevoli contributi di ricerca negli anni passati. Grazie a due tranche di finanziamenti Prin - nel 2005 e del 2007 - sono riuscite a unire le proprie forze e a portare avanti questo ambizioso lavoro sulle vicende dei nostri soldati nell'area balcanica durante la Seconda Guerra Mondiale.

L'obiettivo del progetto era realizzare una ricerca che affrontasse complessivamente la vicenda dei soldati del Regio Esercito presenti in una vasta area: dalla Slovenia a pochi chilometri delle coste turche, passando per la Dalmazia, l'Erzegovina, il Montenegro, la Grecia continentale e quella insulare. Si tratta di un'area molto estesa, in cui, alla vigilia dell'8 settembre 1943, erano presenti ben 650mila uomini che erano stati dislocati in un intricatissimo scenario, in cui la presenza italiana si andava a incrociare con la fitta trama di violenti conflitti etnici e ideologici che contrassegnano questi territori.

Le vicende affrontate dal volume sono di estrema complessità poiché si affrontano temi emersi negli ultimi anni a proposito degli studi sul fascismo e la seconda guerra mondiale: la fine del regime e l'armistizio italiano del $1943^{1}$, le guerre fasciste e le politiche imperialiste italiane in area mediterranea ${ }^{2}$, l'occupazione e i crimini italiani

1 Cfr. AGA ROSSI, Elena, Una Nazione allo sbando. L'armistizio italiano del 1943 e le sue conseguenze, Bologna, Il Mulino, 2003 e MELLONI, Alberto (a cura di), Otto settembre 1943. Le storie e le storiografie, Reggio Emilia, Diabasis, 2005.

2 Cfr. ROCHAT, Giorgio, Le guerre italiane 1935-1943. Dallimpero d'Etiopia alla disfatta, Torino, Einaudi, 2005 e RODOGNO, Davide, Il nuovo ordine mediterraneo. Le politiche di occupazione dell'Italia fascista (1940-1943), Torino, Bollati Boringhieri, 2003. 
nei Balcani'3, i numerosi casi di resistenza dei militari ai soprusi tedeschi dopo l'8 settembre lontano dall'Italia 4 e il successivo internamento dei nostri soldati in Germania 5 , le disavventure dei nostri connazionali rimasti in questi territori nell'ultimo scorcio del conflitto ${ }^{6}$ e i tentativi dei governi italiani del periodo di transizione alla Repubblica di tornare ad avere una, seppur minima, credibilità internazionale dopo l'occupazione e la sconfitta7.

Il libro è introdotto da due capitoli incentrati sull'occupazione dell'area nel corso del conflitto e, in particolare, sulla controversa vicenda dei rapporti tra il nostro esercito e le forze della resistenza locale a seguito della repressione messa in atto da parte italiana. Queste pagine si basano sia su letteratura scientifica italiana e locale, che su fonti dei nostri archivi militari, corroborate da ricerche su documentazione angloamericana e russa in gran parte dei casi inedita.

L'aspetto dominante di questa monografia è indubbiamente la ricostruzione degli avvenimenti riguardanti i reparti presenti nell'area dopo l'annuncio dell'Armistizio. Il lavoro di ricerca è presentato attraverso una suddivisione che rispecchia la dislocazione geografica delle trentacinque divisioni italiane che si ritrovarono intrappolate tra Jugoslavia, Albania e Grecia e si basa su di un'ampia mole di documenti d'archivio provenienti in gran parte dall'Ufficio Storico dello Stato Maggiore Esercito. Nello specifico, sono risultate di particolare importanza le relazioni degli ufficiali redatte al

3 Cfr. Il numero monografico 'Hidden pages of Italian history' del Journal of Modern Italian Studies, 3/2004; OLIVA, Gianni, «Si ammazza troppo poco». I crimini di guerra italiani. 194043, Milano, Mondadori, 2006 e GOBETTI, Eric, L'occupazione allegra. Gli italiani in Jugoslavia (1941-1943), Roma, Carocci, 2007.

4 Sulle vicende dei militari italiani dopo l'armistizio cfr. VALLAURI, Carlo, Soldati. Le forze armate italiane dall'armistizio alla Liberazione, Torino, Utet, 2003; nell'area il caso maggiormente studiato è stato quello di Cefalonia, cfr., ROCHAT, Giorgio, VENTURI, Marcello (a cura di), La divisione Acqui a Cefalonia, Milano, Mursia, 1993 e RUSCONI, Gian Enrico, Cefalonia. Quando gli Italiani si battono, Torino, Einaudi, 2004; negli ultimi anni sono stati realizzati studi anche su altri episodi nello scacchiere balcanico come INSOLVIBILE, Isabella, Kos, 1943-48. La strage la storia, Napoli, Edizioni Scientifiche Italiane, 2010.

5 Cfr. SCHREIBER, Gerhard, I militari internati nei campi di concentramento del Terzo Reich, 1943-19 45. Traditi, disprezzati, dimenticati, Roma, Ufficio SME, 1992 [Ed. originale: Die italienischen Militarinternierten in deutschen Machtbereich : 1943 bis 1945, Verraten, Verachtet, Vergessen, Munchen-Wien, Oldenbourg, 1990]; HAMMERMANN, Gabriele, Gli internati militari italiani in Germania. 1943-1945, Bologna, Il Mulino, 2004 [Ed. originale: Zwangsarbeit für den Verbündeten : Die Arbeits- und Lebensbedingungen der italienischen Militärinternierten in Deutschland 1943-1945, Tübingen, Niemeyer, 2002] e AVAGLIANO, Mario, PALMIERI, Marco (a cura di), Gli internati militari italiani. Diari e lettere dai lager nazisti, 1943-1945, Torino, Einaudi, 2009.

6 Cfr. DI SANTE, Costantino, Nei campi di Tito. Soldati, deportati e prigionieri di guerra italiani in Jugoslavia (1941-1952), Verona, Ombre Corte, 2007.

7 FOCARDI, Filippo, KLINKHAMMER, Lutz, «La difficile transizione. L'Italia e il peso del passato», in ROMERO, Federico, VARSORI, Antonio (a cura di), Nazione, interdipendenza, integrazione. Le relazioni internazionali dell'Italia (1917-1989), vol. I, Roma, Carocci, 2005, pp. 113-129. 
momento del rientro in Italia per dar conto del loro comportamento successivamente all'8 settembre. Questa documentazione è stata messa a confronto con la memorialistica, precedenti studi e testimonianze orali raccolte dalle stesse autrici.

Nell'ultima parte del volume, attraverso una base documentaria di natura militare e diplomatica di provenienza soprattutto italiana e inglese, si analizzano: le traversie dei militari finiti prigionieri dei tedeschi, quelle di coloro che si ritrovano nelle mani delle forze partigiane - in gran parte di orientamento comunista - e delle diverse migliaia di nostri connazionali che, in seguito all'arrivo dell'Armata Rossa nei territori dell'Europa orientale già occupati dalla Wehrmacht, proseguirono la detenzione in mano sovietica. Queste vicende si sovrapposero alla complessa partita diplomatica tra i governi italiani di transizione, gli alleati alla vigilia del profilarsi della divisione in blocchi del continente europeo al termine del conflitto. La disputa si sarebbe protratta per diversi anni, durante i quali decine di migliaia di nostri connazionali vissero momenti terribili, mentre alcune centinaia di criminali di guerra italiani - in maggioranza ufficiali di grado superiore - riuscirono ad evitare i processi.

Leggendo il libro si ha da subito l'impressione che lo studio sia una diretta filiazione del fondamentale lavoro di Elena Aga Rossi sull'armistizio del 1943, Una nazione allo sbando. Sorretta dall'idea che l'8 settembre rappresenti un drammatico spartiacque nella vicenda del nostro paese, questa ricerca si distingue chiaramente per un'accurata ricostruzione dei militari italiani, che furono letteralmente abbandonati al proprio destino all'annuncio dell'armistizio. Nello scacchiere balcanico questo dramma va compreso e analizzato avendo ben presente che, prima di diventare vittime, i nostri militari occuparono quest'area, spesso in maniera brutale e violenta, interpretando il ruolo di braccio armato dell'aggressiva politica estera fascista.

Con questa consapevolezza e con la conoscenza degli intricati conflitti etnicopolitici locali che si scatenarono con l'invasione delle forze dell'Asse nel 1941, le autrici hanno fatto luce sulle diversificate e controverse vicende che coinvolsero i nostri militari all'annuncio dell'armistizio. La costante di tutte le vicissitudini dei soldati italiani fu sicuramente l'impreparazione e l'indecisione dei comandi italiani, che si abbinò alla rapidità e all'aggressività dimostrata immediatamente dalle forze tedesche nel mettere fuori combattimento i reparti del Regio Esercito. Emerge con forza come la risposta dei singoli reparti non fu univoca e il comportamento dei numerosissimi presidi italiani colti di sorpresa dall'armistizio possa essere condotto ad alcune principali dinamiche. 
Questi soldati, inquadrati in un'organizzazione in cui si era abituati a «obbedire, non a prendere iniziative ${ }^{8}$, si trovarono costretti a compiere repentinamente scelte difficili. Il merito delle autrici è stato di riuscire ad entrare nel dettaglio di una così diversificata gamma di avvenimenti. In particolare, viene chiaramente evidenziato come si sia rivelato decisivo, in buona parte dei casi, il carisma degli ufficiali inferiori, che, di fronte all'insipienza dei comandi superiori, rappresentarono gli unici riferimenti per la truppa9. Questi ufficiali furono chiamati a prendere decisioni in un momento in cui non solo si sancì la sconfitta italiana, ma fu un intero mondo di valori a crollare ${ }^{10}$.

Alcuni singoli episodi di quel settembre ' 43 sono drammaticamente paradigmatici, al di là delle diverse opzioni in campo e delle specificità dei diversi contesti: risalta a tal proposito la vicenda del maggiore Bruno Koch, che si trovava con la divisione Bergamo in Dalmazia, e che il 12 si uccise di fronte alle umiliazioni e alle pressioni a cui furono sottoposti gli uomini del suo reparto sia da parte tedesca che partigiana ${ }^{11}$.

Il merito delle autrici è di aver saputo inserire la tragedia dell'8 settembre nel contesto "a parte" dei Balcani, delineando però un quadro rivelatore della contraddittoria vicenda di un paese, l'Italia, che dalla dittatura fascista, attraverso la sconfitta bellica, si avviò alla democrazia. Questa fase di passaggio poté avviarsi grazie agli eventi dell'estate del 1943, ma con cui in realtà il paese non riuscì a fare i conti fino in fondo, sia per via delle scelte della classe dirigente post-fascista e, nel dopoguerra, della sedimentazione della memoria collettiva su queste vicende. L'hanno dimostrato in maniera emblematica i casi dei criminali di guerra italiani mai processati, che avrebbero potuto invece rendere giustizia ai tanti militari che avevano fatto il proprio dovere. Si è preferito invece l'oblio, contribuendo a tenere aperte ferite che hanno diviso la nostra società negli anni seguenti. L'evidente risultato, hanno giustamente sottolineato le autrici, è stato quello di non essere mai giunti ad una completa e sincera

\footnotetext{
8 AGA ROSSI, Elena, GIUSTI, Maria Teresa, Una guerra a parte. I militari italiani nei Balcani 1940-1945, Bologna, Il Mulino, 2011, p. 15.

9 Il ruolo degli ufficiali emerge sia nell'orientare la scelta nel collaborare con i tedeschi, cfr. ibidem. pp. 109 e 119, che in senso opposto, cfr. ibidem, p. 184.

${ }^{10}$ Il riferimento è al titolo dell'epistolario di Giovanni Pirelli Cfr. PIRELLI, Giovanni, Un mondo che crolla : lettere 1938-1943, Milano, Archinto, 1990 ricordato in AGA ROSSI, Elena, GIUSTI, Maria Teresa, Una guerra a parte, cit., p. 14.

${ }_{11}$ Cfr. ibidem, p. 15 e p. 145. Il giorno dopo il suicidio di Koch, un altro ufficiale italiano compì lo stesso gesto: il colonnello Giovanni Faccin. Faccin si trovava in Italia, a Potenza, presso il comando della $7^{\wedge}$ Armata; come Koch fu abbandonato dai suoi superiori nelle ore successive all'annuncio dell'armistizio e fu costretto ad assumersi la responsabilità del comando e dei soldati da lui dipendenti. Il suicidio arrivò dopo cinque giorni di estrema pressione psicologica, mentre era nascosto in una galleria con i suoi uomini. Il colonnello era nell'impossibilità di eseguire e dare ordini, sotto la minaccia delle bombe anglo-americane e dei tedeschi in ritirata. Ho approfondito la vicenda nella mia tesi di dottorato: Mezzogiorno 1943. Soldati allo sbando, Istituto Italiano di Scienze Umane, Firenze-Napoli, a.a. 2010/2011, pp. 62-105; l'episodio è menzionato anche in AGA ROSSI, Elena, Una nazione allo sbando, cit., pp. 144-145.
} 
pacificazione interna, che, al contrario, sarebbe potuta scaturire da una chiara volontà di fare subito chiarezza e rendere evidenti le responsabilità di un periodo così tragico della storia italiana ${ }^{12}$.

Si può affermare che questo volume è, a buon diritto, apprezzabile anche perché arriva ad essere pubblicato in un momento storiograficamente maturo per questi temi, raccogliendo i frutti di un terreno che è stato meritoriamente dissodato da diversi altri lavori usciti negli ultimi anni, che hanno contribuito a interrompere i lunghi decenni di silenzio su questi argomenti.

In conclusione, il libro di Elena Aga Rossi e Maria Giusti non emetterà una sentenza definitiva su questi temi, ma è sicuramente un notevole lavoro di ricerca, sintesi e comprensione, che contribuisce a fare un'ulteriore e, ritengo, decisiva chiarezza su questo periodo, dopo tanti studi che, seppur scientificamente notevoli, sono riusciti solo parzialmente a tenere assieme le questioni affrontate in quest'opera.

${ }^{12}$ Cfr. AGA ROSSI, Elena, GIUSTI, Maria Teresa, Una guerra a parte, cit., p. 439. 


\section{${ }^{*}$ L'autore}

Mario De Prospo è dottore di ricerca $(\mathrm{PhD})$ in Storia Contemporanea, titolo conseguito presso L'Istituto Italiano di Scienze Umane, SUM (Firenze, Napoli). Collabora con il dipartimento di discipline Storiche "Ettore Lepore" dell'Università degli Studi di Napoli Federico II ed è specializzando della Scuola Speciale per Archivisti e Bibliotecari presso l'Università degli Studi di Roma La Sapienza. Attualmente è consulente archivistico presso il Fondo Internazionale per lo Sviluppo Agricolo (IFAD), agenzia ONU con sede a Roma. Le sue ricerche affrontano la storia militare, sociale e delle istituzioni dell'Italia nella transizione dal fascismo alla Repubblica. Ha curato il volume Avellino 1943: le bombe, la tragedia, la memoria (Avellino, Mephite, 2012). Sono di prossima pubblicazione i saggi Reconstructing the army of a collapsed nation: the Kingdom of the South of Italy (September 1943 - March 1944 ) su «The Journal of Modern Italian Studies» e Mezzogiorno 1943. Esercito e società, sui «Quaderni della Fondazione Luigi Salvatorelli».

URL: < http://studistorici.com/progett/autori/\#DeProspo >

\section{Per citare questo articolo:}

DE PROSPO, Mario, «Recensione: Elena AGA ROSSI, Maria Teresa GIUSTI, Una guerra a parte. I militari italiani nei Balcani 1940-1945, Bologna, II Mulino, 2011, 660 pp.», Diacronie. Studi di Storia Contemporanea: La satira fa storia. Eventi, pratiche, linguaggi, 29/10/2012,

URL:< http://www.studistorici.com/2012/10/29/de-prospo_numero_11/ >

Diacronie Studi di Storia Contemporanea $\beta$ ww.diacronie.it

Risorsa digitale indipendente a carattere storiografico. Uscita trimestrale. redazione.diacronie@hotmail.it

Comitato di redazione: Marco Abram - Giampaolo Amodei - Jacopo Bassi - Luca Bufarale - Gianluca Canè - Alessandro Cattunar - Alice De Rensis - Barbara Galimberti - Deborah Paci - Fausto Pietrancosta - Matteo Tomasoni - Luca Zuccolo 